\title{
Measurement of Esophagogastric Junction Distensibility May Assist in Selecting Patients for Endoluminal Gastroesophageal Reflux Disease Surgery: Author's Reply
}

TO THE EDITOR: We thank the author for his interest in our manuscript. ${ }^{1,2}$ During the transoral incisionless fundoplication (TIF) procedure, a retractor is anchored at the esophagogastric junction (EGJ) after which tension is applied to advance the EGJ caudally. ${ }^{3,4}$ Therefore, we agree that lengthening of the EGJ is a substantial mode of action of the TIF procedure. In addition, transmural placement of polypropylene fasteners creates an endoluminal fundoplication through permanent serosa-to-serosa or serosa-to-muscularis fusion with tightening of the distal esophagus, which decreases EGJ diameter and distensibility. Therefore, we believe that EGJ distensibility can be an important parameter for evaluation of endoluminal gastroesophageal reflux disease (GERD) surgery like the TIF procedure, although we agree that the immediate decrease in EGJ distensibility after the TIF procedure might be the consequence of postoperative edema. ${ }^{7}$

It is important to note that consensus is lacking with regard to analysis of parameters obtained from EndoFLIP measurements. According to the most widely accepted approach, EGJ distensibility is assessed based on the narrowest cross sectional area and corresponding intra-bag pressure. Standardized methods to evaluate the change in EGJ length with the EndoFLIP technique are still to be defined. Therefore, we anticipate that development of more extensive biomechanical measures will result in more accurate interpretation of EndoFLIP data.

In the present study, we used the EndoFLIP technique in the preoperative diagnostic work-up to predict postoperative outcome. Recent studies highlight that the EndoFLIP technique could be used as an intra-operative quality tool during either antireflux or achalasia surgery. ${ }^{8,9}$ Teitelbaum et $\mathrm{al}^{10}$ described that the EndoFLIP technique is able to guide laparoscopic Heller my- otomy and peroral esophageal myotomy to obtain an ideal postoperative EGJ distensibility with regard to both postoperative achalasia and reflux symptoms. In addition, Perretta et al $^{8}$ provided observational data about the change in EGJ distensibility and diameter during different stages of laparoscopic Nissen fundoplication. In the future, the EndoFLIP method could potentially be used during laparoscopic fundoplication to prevent creation of a hypercompetent valve with associated postoperative symptoms (eg, dysphagia or gas bloating). ${ }^{8}$

It therefore appears that the EndoFLIP technique has considerable potential as an intra-operative tool. On the other hand, currently available methodologies limit its role with regards to the assessment of EGJ distensibility in the preoperative diagnostic work-up or in post-procedure evaluation of patients undergoing endoluminal GERD surgery like TIF. Additional research is necessary to define the role of the EndoFLIP technique for both the preoperative work-up and tool for intraoperative calibration.

Fabiënne G M Smeets, Daniel Keszthelyi,
Ad A Masclee, and José M Conchillo
Department of Gastroenterology and Hepatology, Maastricht University
Medical Center, Maastricht, the Netherlands

1. Smeets FG, Keszthelyi D, Bouvy ND, Masclee AA, Conchillo JM. Does measurement of esophagogastric junction distensibility by EndoFLIP predict therapy responsiveness to endoluminal fundoplication in patients with gastroesophageal reflux disease? J Neurogastroenterol Motil 2015;21:255-264.

2. O'Dea J. Measurement of esophagogastric junction distensibility may assist in selecting patients for endoluminal Gastroesophageal Reflux Disease Surgery. J Neurogastroenterol Motil 2015;21:448449. 
3. Bell RC, Freeman KD. Clinical and $\mathrm{pH}$-metric outcomes of transoral esophagogastric fundoplication for the treatment of gastroesophageal reflux disease. Surg Endosc 2011;25:1975-1984.

4. Bell RC, Cadière GB. Transoral rotational esophagogastric fundoplication: technical, anatomical, and safety considerations. Surg Endosc 2011;25:2387-2399.

5. Hoppo T, Immanuel A, Schuchert M, et al. Transoral incisionless fundoplication 2.0 procedure using EsophyX for gastroesophageal reflux disease. J Gastrointest Surg 2010;14:1895-1901.

6. Hoppo T, McMahon BP, Witteman BP, et al. Functional lumen imaging probe to assess geometric changes in the esophagogastric junction following endolumenal fundoplication. J Gastrointest Surg 2011;15:1112-1120.

7. Jobe BA, O'Rourke RW, McMahon BP, et al. Transoral endoscopic fundoplication in the treatment of gastroesophageal reflux disease: the anatomic and physiologic basis for reconstruction of the esophagogastric junction using a novel device. Ann Surg 2008;248: 69-76.

8. Perretta S, McAnena O, Botha A, et al. Acta from the EndoFLIP® Symposium. Surg Innov 2013;20:545-552.

9. Teitelbaum EN, Boris L, Arafat FO, et al. Comparison of esophagogastric junction distensibility changes during POEM and Heller myotomy using intraoperative FLIP. Surg Endosc 2013;27:45474555 .

10. Teitelbaum EN, Soper NJ, Pandolfino JE, et al. Esophagogastric junction distensibility measurements during Heller myotomy and POEM for achalasia predict postoperative symptomatic outcomes. Surg Endosc 2015;29:522-528.

\section{Conflicts of interest: None.}

